\title{
APOE is a Prognostic Biomarker and Correlates With Immune Infiltrates in Papillary Thyroid Carcinoma
}

Xu Lin

Hebei North University https://orcid.org/0000-0002-3118-053X

Wen-Jing Zhang

Department of Histology and Embryology, Hebei North University, Zhangjiakou, 075000, China

Gang Xue

Department of Otorhinolaryngology Head and Neck Surgery, Hebei North University, Zhangjiakou, 075000, China

Jing-Fang Wu ( $\nabla$ wjfxg@163.com )

Department of Histology and Embryology, Hebei North University, Zhangjiakou, 075000, China

\section{Liu-ya Chao}

Department of Otorhinolaryngology Head and Neck Surgery, Hebei North University, Zhangjiakou, 075000, China

\section{Research}

Keywords: papillary thyroid carcinoma, APOE, immune infiltrates, biomarker

Posted Date: January 7th, 2021

DOl: https://doi.org/10.21203/rs.3.rs-139868/v1

License: (c) (i) This work is licensed under a Creative Commons Attribution 4.0 International License. Read Full License

Version of Record: A version of this preprint was published at Journal of Cancer on January 1st, 2022. See the published version at https://doi.org/10.7150/jca.63545. 


\section{Abstract}

Background: Recent research showed that abnormal lipid metabolism was associated with cancers. As one of the genes that can regulate the level of lipid metabolism, abnormal APOE expression was associated with carcinogenesis. However, the biological role of APOE in papillary thyroid carcinoma (PTC) remains to be determined.

Methods: ONCOMINE, GEPIA, UALCAN, STRING, GeneMANIA, LinkedOmics, GSCALite, TISIDB, EPIC and TIMER were utilized to achieve comprehensively bioinformatics analysis of APOE in this study. And the immunohistochemical staining of APOE was used to verify the predicted results.

Results: The mRNA level and protein level of APOE of PTC tissues were significantly elevated in TCGA cohort and Shanghai cohort. And APOE expression was positively correlated with the pathological stage and lymph node metastasis in PTC. PTC patients with low mRNA level of APOE were associated with a bad prognosis. The functions of APOE co-expressed genes were mainly enriched in adaptive immune response, protein-lipid complex subunit organization, actin cytoskeleton reorganization, cell chemotaxis, protein activation cascade and transcriptional misregulation in cancer. APOE level was significantly correlated with tumor-infiltrating cells (B cells, CD8+ T cells, neutrophils, and dendritic) and immune biomarkers in PTC.

Conclusions: APOE is a potential independent biomarker for PTC and APOE expression is positively correlated with immune cell infiltration in PTC.

\section{Background}

Thyroid Papillary Carcinoma (PTC) is the most common subtype of thyroid cancer, accounting for about $80 \%$ and its incidence, lymph node metastasis rate, disease burden, recurrence rate and mortality rate have increased significantly in recent years $(1,2)$. As the most common endocrine tumor, PTC has a tendency to occur lymph node metastasis in the early stages of cancer, and $20-30 \%$ of PTC patients have already suffered from cancer cell lymph node involvement at the time of diagnosis $(3,4)$. Although some mechanisms have been clarified, including growth factors, ionizing radiation, iodine intake, gender, and genetic factors, these have not yet specifically clarified the mechanism of PTC occurrence and cannot be used as a specific target for clinical treatment for PTC. At the same time, the tumor recurrence rate, distant metastasis rate and mortality rate will be significantly increased of the PTC patients with lymph node metastasis compared to those without metastasis $(5,6)$. Thus, excavation a novel biomarker for assisting screening diagnosis, predicting PTC prognosis and blocking PTC lymph node metastasis.

Apolipoprotein E (ApoE) can be synthesized by various tissues such as liver, intestine, adrenal gland, kidney, lung, spleen, ovary and brain. Meanwhile, APOE not only plays the role of lipid transport, storage, utilization and excretion in different organizations, it can also participate in the regulation of various diseases such as Schizophrenia, coronary heart diseas $₫$ diabetic nephropathies, Alzheimer's disease, and malignant tumor, and play a variety of biological activities. Tavazoie et al pointed out that APOE may be 
involved in lymphocyte-mediated immune regulation, and that overexpression of APOE promotes the biological processes of various malignant tumors $(7,8)$. Geng et al suggested that APOE modulates the immune response by inhibiting antigen-activated lymphocytes (9). Sakashita $\mathrm{K}$ et al investigated the relationship between APOE and gastric cancer by immunohistochemistry and RT-PCR in clinical samples of tumor tissue and corresponding normal tissue from patients with gastric cancer, and the overexpression of APOE in gastric cancer tissues exhibited stronger malignant invasiveness compared to cancer tissues with low APOE expression (10). And over-expression of APOE significantly promotes the ability of invasion and lymph node metastasis of gastric cancer cells (10). There is increasing evidence that APOE is abnormally expressed in a variety of solid cancers and may be a biomarker for many tumors, including bladder cancer and ovarian cancer. However, the function of APOE in PTC are still not clarified.

In this study, we analyzed the expression of APOE in PTC using Oncomine database and TCGA database, and then analyzed its clinical significance, prognostic value and immune function by various analysis tools. Our results may provide more references for further study of APOE in PTC.

\section{Methods}

\section{TCGA data source and tissue samples}

The data for 512 PTC and 18 normal thyroid tissue samples were acquired from The Cancer Genome Atlas (TCGA, https://tcga-data.nci.nih.gov/tcga/) database. The PTC tissue microarray (HThy-Pap120CS01), including 58 PTC tissues and 58 matched tumor-adjacent tissues, was bought from Shanghai Outdo Biotech CO. , LTD. All 58 patients were diagnosed as PTC by experienced pathologists without any treatment before surgery.

\section{GEPIA}

GEPIA (www.gepia.cancer-pku.cn/index.html) is not only a website that can quickly and easily identify the expression of specific genes in certain cancers, but also provides survival analysis, clinical information analysis and correlation analysis of specific genes. In our study, the expression analysis and prognostic analysis of APOE was performed using TCGA PTC samples with a p-value $<0.05$.

\section{Oncomine analysis}

Oncomine, an efficient data mining platform, can analyze the expression of genes in the most common cancer at the transcription level (11). The gene expression level of APOE was analyzed by Oncomine database according to the data of PTC mRNA levels of cancer vs normal patient datasets.

\section{The Human Protein Atlas}

The Human Pathology Atlas project (https ://www.proteinatlas.org) is a website that can help researchers to provide immunohistochemistry and immunofluorescence results of specific genes in cancer and 
normal tissues (12). In this experiment, we compared the immunohistochemical staining results of APOE gene in papillary thyroid carcinoma and normal thyroid tissue.

\section{UALCAN analysis}

The UALCAN database is a comprehensive database based on OMICS data that allows users to quickly identify gene expression, clinical information, and oncogenes in a variety of solid tumors (13). In this study, we analyzed APOE expression by gender, age, with/without lymph node metastasis, and clinical stage with a p-value $<0.05$.

\section{STRING and GeneMANIA}

STRING (http://www.string-db.org/) is an online tool that can be used to analyze protein-protein interaction networks of multiple species (14). Similar to STRING, GeneMANIA (http://www.genemania.org) is an online database that can identify specific gene interaction networks by collecting published data in multiple databases (15). At the same time, it can also show the colocalization, co-expression, and correlation between these interacting genes. After obtained the genes from networks using STRING, we used the GeneMANIA analysis website to make a preliminary exploration of the association and role of these genes.

\section{LinkedOmics and GSCALite}

LinkedOmics (www.linkedomics.org) is a complex and powerful platform that can help researchers to accurately dig up key genes from massive data by analyzing various cancer data in the TCGA database (16). GSCALite邓www.bioinfo.life.hust.edu.cn/web/GSCALite/》 is a powerful platform for multi-group analysis, which can directly visualize the signal pathways that may play a role in gene groups in various solid malignancies according to TCGA data (17). We extracted TCGA PTC sample and the data of 502 PTC patients were acquired. We first analyzed the genes that showed a significant correlation with APOE through the LinkFinder module. Then, the biological process, pathway, kinase, miRNA and transcription factor-target analysis of APOE was performed with a p-value $<0.05$. And GSCALite provided pathway activity analysis with the TCGA PTC samples once more.

\section{Immune infiltration analysis}

TIMER (www.cistrome.shinyapps.io/timer/), a genetic immunoassay platform, can directly perform immunological analysis on specific genes in certain cancer and visualize the results for researchers(18). At the beginning of the article, we analyzed the expression of APOE gene in various cancers and normal tissues through the "DiffExp module" in TIMER. Then, the correlation between APOE and the level of immune infiltrating cells in PTC and the relationship between APOE gene and biomarkers of immune cells were conducted by "gene module" and "correlation module", respectively $(19,20)$. TISIDB (http://cis.hku.hk/TISIDB/) uses high-throughput analysis of genetic data to identify and predict the association of specific genes with tumor immune cell infiltration (21). To reconfirm whether APOE is involved in regulating immune infiltration in PTC, we used TISIDB to investigate the relationship between 
APOE expression and immunoinhibitors, immunostimulators, MHC molecules and TILs. $p<0.05$ was considered statistically significant.

The EPIC (https://gfellerlab.shinyapps.io/EPIC_1-1/) application can help researchers to assess the proportion of immune cells and cancer cells under different expression conditions of specific genes. To evaluate the effect of APOE expression on immune cells, we divided 512 PTC samples in TCGA into two groups (APOE high expression group and APOE low expression group), and then imported the data into the EPIC application tool for analysis. Finally, the analysis results were statistically analyzed and visualized using GraphPad Prism 7 software with p-value $<0.05$.

\section{Immunohistochemical (IHC) staining and evaluation}

Immunochemical staining of APOE was conducted using mouse monoclonal anti-APOE antibody (1: 100, catalog number RLT0273, Ruiying Biological, China). The results of APOE negative or positive staining in PTC were evaluated by two experienced pathologists and were determined as follows. The overall APOE IHC score grading from 1 to 5 was evaluated according to the semi-quantitative immunoreactive score (IRS) scale of Remmele(22).

\section{Results}

\section{The levels of APOE expression in THCA and other cancers}

Gene expression analyses using the TIMER database based on TCGA data showed that APOE mRNA levels were significantly higher in breast cancer (BRCA), Esophageal carcinoma $\triangle E S C A \rrbracket, H e a d$ and Neck squamous cell carcinoma (HNSC), Liver hepatocellular carcinoma (LIHC), Prostate adenocarcinoma (PRAD), Stomach adenocarcinoma (STAD), Thyroid carcinoma (THCA) and Uterine Corpus Endometrial Carcinoma (UCEC) compared with the corresponding normal tissues (Fig.1). To further investigate whether the abnormal expression of APOE affects the occurrence of thyroid cancer, we then evaluated APOE transcription levels from multi-database. As shown in Fig.2a, APOE mRNA expression was significantly upregulated in thyroid cancer than in adjacent normal tissues according to the GEPIA database. Data in the Oncomine database showed that APOE mRNA expression was significantly higher in PTC and ranked within the top $2 \%$ based on mRNA expression (Fig. $1 \mathrm{~b}$ ). IHC staining according to the the Human Protein Atlas database showed that APOE expression was not detected in normal controls, while the moderate APOE expression was showed in PTC tissue (Fig.2c) .

\section{APOE expression correlated with clinicopathological parameters for PTC patients in TCGA cohort and Shanghai cohort}

Next, we used the UALCAN web resource to study the relationship between APOE expression and different clinical characteristics of thyroid cancer. As shown in Fig.3, the expression of APOE was significantly higher in PTC patients than in normal controls with multi-analysis based on gender, age, metastasis 
status and different stages. In particular, APOE mRNA expression in PTC samples was significantly correlated with $₫$-囚stages.

Then, 58 matched tumor and normal samples were immunohistochemically stained to verify whether the expression of APOE protein in the Shanghai cohort was consistent with the TCGA database and The HPA database. We found that in Shanghai cohort, APOE protein expression in PTC samples was significantly increased compared with adjacent tissues: $98.6 \%$ of PTC patients had higher APOE expression levels than in the normal tissues (Fig.5). Besides, the APOE expression in PTC of Shanghai cohort was positively correlated with pathological stage $(p=0.001, r=0.6978)$ and lymph node metastasis $(p=$ $0.001, r=0.7$ ) (Table 1). In order to reveal the relationship between APOE and PTC survival outcome, the survival curve analysis based on TCGA data was analyzed by GEPIA. However, the low APOE expression group had significantly shorter overall survival (Logrank, $p=0.027$ ) compared to the high expression group and APOE expression had no association with disease-free survival(Logrank, $p=0.07)$. To determine the potential diagnostic value of APOE, the ROC curves of APOE were generated in TCGA cohort and Shanghai cohort, respectively (Fig.5). The ROC curve analysis showed that APOE had a satisfactory diagnostic value and the AUC of APOE were 0.7819 and 0.9064 in TCGA cohort and Shanghai cohort, respectively.

\section{Enrichment analysis of APOE in PTC}

Two PPI networks analysis of APOE were conducted by using STRING and GeneMANIA to explore the potential interactions among the proteins interacted with APOE. 12 nodes and 778 edges were acquired in the PPI network (Fig.6a). The function of these APOE-related genes was associated with posttranslational protein modification, extracellular structure organization, inflammatory response. Results of GeneMANIA suggested that the roles of these APOE-related genes were basically linked to blood microparticle, plasma lipoprotein particle, protein-lipid complex, high-density lipoprotein particle, regulation of plasma lipoprotein particle levels, plasma lipoprotein particle remodeling and protein-lipid complex remodeling (Fig.6b).

To further understand the biological significance of APOE in PTC, LinkedOmics and GSCALite tools were used to explore the APOE co-expression patterns and possible pathways in the TCGA cohort. The results of LinkedOmics platform demonstrated 11534 genes (dark red dots) positively correlated with APOE and 8392 genes (dark green dots) negatively correlated with APOE in PTC (Fig.7a). The top 50 significant genes positively and negatively correlated with APOE in PTC were shown in Fig.7b and Fig.7c , respectively. Moreover, APOC1 (cor=0.9121, p=2.17e-195), APOC1P1 (cor=0.7111, p=2.27e-78), ISYNA1 (cor=0.6479, $p=5.45 e-61$ ) and C7orf61 (cor=0.6777, $p=1.21 \mathrm{e}-68$ ) were the four hub genes most positively correlated with APOE in PTC. Enrichment analysis was also performed. GO items showed that APOE co-expressed genes mainly participated in adaptive immune response, protein-lipid complex subunit organization, artery development, actin cytoskeleton reorganization, cell chemotaxis, amyloidbeta clearance and protein activation cascade, while the activities like ER-nucleus signaling pathway, nucleoside triphosphate metabolic process, Golgi vesicle transport, nucleobase metabolic process, 
mitochondrial respiratory chain complex assembly, coenzyme metabolic process, mitochondrial gene expression and tricarboxylic acid metabolic process were inhibited (Fig.7d). And KEGG pathway items revealed that enrichment in transcriptional misregulation in cancer, staphylococcus aureus infection, cytokine-cytokine receptor interaction, osteoclast differentiation, neuroactive ligand-receptor interaction, allograft rejection, cholesterol metabolism, leukocyte transendothelial migration, inflammatory bowel disease(IBD), natural killer cell-mediated cytotoxicity, and ECM-receptor interaction(Fig.7e). In order to further explore the potential mechanism of the five key genes (APOC1, APOC1P1, ISYNA1, C7orf61 and APOE) and whether these genes function through common cancer pathways, we analyzed them using the GSCALite platform by pathway activity module. As illustrated in Fig.8, APOE participated in the activation of processes such as Apoptosis, Cell Cycle, DNA Damage Response, EMT and Hormone ER, and the inhibition of Hormone AR, PI3K/AKT, RTK and TSC/mTOR signaling pathways.

\section{Regulators of APOE in PTC}

Owing to the significance of APOE in PTC, we further analyzed APOE

networks of kinase, miRNA and transcription factor targets in PTC (Table 2). Only one kinase target of APOE was identified (Kinase_LCK) from the LinkedOmics database. Then, PPI network was constructed to reveal the underlying mechanism of kinase LCK, and showed that the function of these genes in T cell activation, regulation of lymphocyte activation / leukocyte activation, positive regulation of $\mathrm{T}$ cell activation/lymphocyte activation/leukocyte activation/cell activation (Additional file 1: Fig. S1). MIR-323 was enriched by GSEA for APOE co-expressed genes (Table 2). Besides, V\$AP1_C, V\$STAT5B_01, V\$NERF_Q2, V\$NFKAPPAB65_01, RGAGGAARY_V\$PU1_Q6, V\$BACH2_01, V\$NGFIC_01 and V\$LBP1_Q6 were the transcription factor network targets of APOE (Table 2), and the functions of these transcription factors were mostly enriched in JAK-STAT signaling pathway, MAPK signaling pathway, growth hormone receptor signaling pathway and regulation of epithelial cell migration (Additional file 2: Fig. S2).

\section{Association of APOE expression with immune cells and biomarkers}

When we analyzed the role of the APOE in PTC using the LinkedOmics platform, we found that the function of the gene was primarily focused on regulating PTC immune response. This suggested that APOE may be involved in the immunoregulatory process of PTC. Then, TIMER platform was used to further clarify the association between APOE expression and immune cell infiltration. For the correlation between APOE expression and immune-related cells, we found a positive association between APOE expression and $B$ cells (Cor=0.228, $P=4.39 \mathrm{e}-07$ ), CD8+T cells(Cor=0.15, $P=9.30 \mathrm{e}-4$ ), Neutrphils (Cor=0.197, $\mathrm{P}=1.14 \mathrm{e}-05)$ and Dendritic cells(Cor=0.229, $\mathrm{P}=3.58 \mathrm{e}-07$ ) (Fig.9a). Fig.9b showed that positive correlations were acquired between APOE expression and the expression of CD8A, CD8B. For TAM, biomarkers including CCL2, CD68 and IL10 were positively correlated with APOE expression. Similar results were obtained in M1 and M2 Macrophage (INOS(NOS2), IRF5, COX2(PTGS2), CD163, VSIG4, MS4A4A ). Therefore, these results may indicate that APOE overexpression was related to the immunomodulatory process and APOE may be a potential target of immunotherapy of PTC. And multivariate COX regression analyses of APOE were performed. As shown in Table 3, tumor purity was associated with poor outcome 
and $\mathrm{CD} 8+\mathrm{T}$ cells $(\mathrm{HR}=0.000,95 \% \mathrm{Cl}=0.000-0.111)$, macrophages $(\mathrm{HR}=0.000,95 \% \mathrm{Cl}=0.000-0.138)$ and Dendritic $(\mathrm{HR}=8478449.036,95 \% \mathrm{Cl}=0.813-8.844267 \mathrm{e}+13)$ might be independent favorable prognostic indicators of PTC patients.

In order to reveal in more detail whether there was a correlation between APOE expression and lymphocytes and immunomodulators (immunoinhibitors, immunostimulators, and major histocompatibility complex (MHC) molecules), we analyzed it using TISIDB database. Fig.10a showed correlations between APOE expression and immunoinhibitors. The immune inhibitors showed strong correlations with APOE expression including CD160 (Spearman: $\rho=0.401, P<2.2 \mathrm{e}-16$ ), TGFB1 (Spearman: $\rho=0.514, \mathrm{P}<2.2 \mathrm{e}-16)$, LGALS9 (Spearman: $\rho=0.338, \mathrm{P}=6.24 \mathrm{e}-15$ ), and TGFBR1 (Spearman: $\rho=0325, \mathrm{P}=8.14 \mathrm{e}-16)$ in PTC (Fig.10b). For immunostimulators, APOE expression was positively correlated with CD40 (Spearman: $\rho=0.444, P<2.2 e-16$ ), KLRK1 (Spearman: $\rho=0.279, P=$ 1.76e-10), TNFRSF8 (Spearman: $\rho=0.607, P<2.2 \mathrm{e}-16$ ), and TNFSF13B (Spearman: $\rho=0.144, P=$ 0.00115) in PTC (Fig.10c-10d). Fig.10e showed correlations between APOE expression and MHC molecules. And there were positive correlations between APOE expression and HLA-B (Spearman: $\rho=$ $0.153, P=0.000557$ ), HLA-DOA (Spearman: $\rho=0.144, P=0.00113$ ), HLA-DPA1 (Spearman: $\rho=0.117, P=$ 0.00843 ), and TAP1 (Spearman: $\rho=0.126, P=0.00437$ ) in PTC (Fig.10f). Besides, the correlation between APOE expression and tumor-infiltrating lymphocytes (TILs) was shown in Fig.10g, and TILs were positively correlated with APOE expression including Act_b (Spearman: $\rho=0.247, P<1.84 \mathrm{e}-08$ ), Act_CD8 (Spearman: $\rho=0.254, P=6.95 e-09)$, Tcm_CD4 (Spearman: $\rho=0.186, P=2.56 \mathrm{e}-05)$, and Tfh (Spearman: $\rho=0359, \mathrm{P}=5.7 \mathrm{e}-17)$ in PTC (Fig.10h).

Finally, the EPIC application was used to analyze whether APOE expression was related to PTC immune infiltration. Among 512 PTC samples, samples were divided into 2 groups (top 1/2 and low 1/2 APOE expression groups). As shown in Fig. $11, B$ cells $(P=0.002)$, CD8 T cells $(P=0.034)$, Macrophage cells ( $P$ $=0.00083)$ and other cells $(P=0.042)$ were main immune cells affected by different APOE expression.

\section{Discussion}

Previous studies had pointed out that tumor tissue needed more energy to maintain growth than normal tissue. Under this circumstance, cancer cells mainly obtained the energy necessary to maintain growth through abnormal lipid metabolism and aerobic glycolysis. More and more evidences showed that the abnormality of lipid metabolism was closely related to the occurrence of tumor (23). APOE (Apolipoprotein E), which regulated cholesterol transport and plasma protein metabolism in cells, was associated with various tumors, such as breast cancer and nervous system tumors $(24,25)$. At present, there were several literatures pointing out that APOE could promote the growth and metastasis of lung carcinomas and ovarian carcinomas through immunoregulatory and differentiated cell growth $(26,27)$. However, the function of APOE in PTC was still not clarified. In this study, we analyzed the potential immunoregulatory, cancer promoting and molecular mechanisms of APOE by bioinformatics. And the results of the bioinformatics prediction were preliminarily verified by the immunochemical staining of PTC tissue chips. 
First, we extensively analyzed APOE expression in various common solid cancers through the TIMER platform. We found that APOE was more highly expressed in breast cancer, Esophageal carcinoma, head and neck squamous cell carcinoma, liver hepatocellular carcinoma, prostate adenocarcinoma, stomach adenocarcinoma, thyroid carcinoma and uterine corpus endometrial carcinoma than the corresponding adjacent tissues. Next, we detected the expression levels of APOE mRNA and protein expression level in PTC. The results from TCGA, Oncomine database and the HPA database showed that APOE mRNA and protein levels were overexpressed in PTC. And APOE mRNA levels were significantly correlated with patients' gender, age, metastasis, clinical stage, and overall survival rate of PTC patients according to TCGA data. Then we performed immunohistochemical staining on the PTC tissue chip to verify whether the results were consistent with the predicted results. The results showed that APOE expression in Shanghai cohort was positively correlated with pathological stage and lymph node metastasis, which was consistent with the predicted result. Some scholars pointed out that the expression level of APOE in the serum of breast cancer patients increased significantly, and these patients were more prone to metastasis (28). And consistent with the results of Xu et al., we found that APOE can also be considered as a prognostic risk factor of PTC (28). Therefore, APOE may play a crucial role in PTC.

We then explored top-50genes, which may interact with APOE, and analyzed their potential roles in a variety of ways. The results of functional enrichment analysis indicated that the functions of APOE and APOE-related genes are mainly involved in post-translational protein modification, adaptive immune response, extracellular structure organization, inflammatory response, blood microparticle, plasma lipoprotein particle, protein-lipid complex, high-density lipoprotein particle, regulation of plasma lipoprotein particle levels, plasma lipoprotein particle remodeling, protein-lipid complex remodeling, protein-lipid complex subunit organization, artery development, actin cytoskeleton reorganization, cell chemotaxis, amyloid-beta clearance and protein activation cascade. And KEGG pathway items revealed that APOE was involved in transcriptional misregulation in cancer, activation of multiple signaling pathways processes such as Apoptosis, Cell Cycle, DNA Damage Response, EMT and Hormone ER, and the inhibition of Hormone AR, PI3K/AKT, RTK and TSC/mTOR signaling pathways. Chen et al pointed out that APOE mRNA expression increased more than 10-fold in ovarian cancer, and that APOE knockdown in OVCAR3 cells could lead to arrested cell growth and induce cell apoptosis (27). Previous studies had shown that elevated cholesterol levels can increase the risk of cancer. Consistent with our findings, Ben pointed out that especially in breast cancer, APOE can inhibit the proliferation and migration of tumor cells by regulating cholesterol metabolism, and APOE can be used as a target to improve prognosis (29). Moreover, APOE can regulate cancer progression by regulating lipid metabolism via TGF $\beta, E M T, E R$ signaling pathways $(29,30)$. Therefore, these results indicated that the function of the APOE gene may be related to cancer and cancer-related pathways, and APOE may mediate the occurrence of PTC.

Kinases, especially tyrosine Kinase, played an important role in regulating cell differentiation and growth under physiological conditions (31). APOE gene expression can be regulated by miRNA and transcription factors through its own unique regulatory mechanisms $(32,33)$. In this experiment, we found that the kinase and miRNA that regulate APOE are LCK, which belong to Src Family Tyrosine Kinase (PKTs) and miRNA-323, respectively. And the functions of Kinases, miRNAs and transcription factors that regulated 
APOE were mainly enriched in T cell activation, regulation of lymphocyte activation/leukocyte activation,

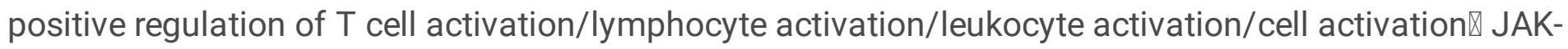
STAT signaling pathway, MAPK signaling pathway, growth hormone receptor signaling pathway and regulation of epithelial cell migration. These results indicated that APOE may affect immune regulation through multiple signaling pathways to promote tumorigenesis of PTC via these three regulators.

Accumulating evidences indicated that APOE can participate in the immune regulation of various diseases $(7,8,34,35)$. Tavazoie et al pointed out that APOE can regulate immunity through myeloidderived suppressor cells (MDSC) accumulation in cancers (7). Moreover, APOE-related signalings in cancers can participate in regulation of immunity (7). In this study, the correlation between APOE level and immune infiltration cells were analyzed, which suggested that high APOE was associated with immune infiltration and we found a positive association between the expression of APOE and the infiltration of B cells, CD8+T cells, Neutrophils, Dendritic cells. Currently, immunotherapy has become one of the most promising strategies in cancer treatment. In this experiment, immunoinhibitors, immunostimulators of APOE were analyzed. The results showed that CD160, TGFB1, LGALS9, TGFBR1, CD40, KLRK1, and TNFRSF8 may serve as APOE immunomodulatory targets. Thus, APOE can not only be used as a diagnostic factor for PTC, but also as a potential target for immunotherapy.

In summary, our results suggest that APOE is a potential independent biomarker for PTC and APOE expression is positively correlated with immune cell infiltration in PTC.

\section{Declarations}

\section{Ethics approval and consent to participate}

Written informed consent was obtained from participants. Ethical permission for the study was granted by the Ethics Committee at Hebei North University.

\section{Acknowledgements}

Special thanks to the Morphology Experiment Center of Hebei North College for their extensive help.

\section{Authors' contributions}

Gang Xue and Jing-Fang Wu designed the study. Wen-Jing Zhang analyzed the data. Xu Lin wrote this article. Xu Lin and Liu-ya Chao was responsible for for editing and submit the manuscript.

\section{Availability of data and materials}

The datasets generated and/or analyzed during the current study are available from the corresponding author on reasonable request.

\section{Consent for publication}


Consent for publication was obtained from included participants.

\section{Competing interests}

The authors declare that the research was conducted in the absence of any commercial or financial relationships that could be construed as a potential conflict of interest.

\section{Funding}

This work was supported by Hebei Provincial Department of Finance Specialist Capacity Building and Specialist Leadership Program (Grant NO: 361009); Hebei Provincial Natural Science Foundation Project (Grant NO: H201840505); Hebei North University 2019 Basic Research Business Expenses Project (Grant NO: JYT2019015).

\section{Competing interests}

The authors declare that they have no competing interests.

\section{References}

1. James BC, Mitchell JM, Jeon HD, Vasilottos N, Grogan RH, Aschebrook-Kilfoy B. An update in international trends in incidence rates of thyroid cancer, 1973-2007. Cancer Causes Control. (2018), 29:465-473.

2. Cabanillas ME, McFadden DG, Durante C. Thyroid cancer. Lancet. (2016), 388:2783-2795.

3. Iorio MV, Croce CM. MicroRNA dysregulation in cancer: diagnostics, monitoring and therapeutics. A comprehensive review. EMBO Mol Med. (2012), 4:143-159.

4. Haugen BR. 2015 American Thyroid Association Management Guidelines for Adult Patients with Thyroid Nodules and Differentiated Thyroid Cancer: What is new and what has changed? Cancer. (2017), 123:372-381.

5. Pourseirafi S, Shishehgar M, Ashraf MJ, Faramarzi M. Papillary Carcinoma of Thyroid with Nasal Cavity Metastases: A Case Report. Iran J Med Sci. (2018), 43:90-93

6. Ullmann TM, Gray KD, Moore MD, Zarnegar R, Fahey TJ, 3rd. Current controversies and future directions in the diagnosis and management of differentiated thyroid cancers. Gland Surg. (2018), 7:473-486.

7. Tavazoie MF, Pollack I, Tanqueco R, Ostendorf BN, Reis BS, Gonsalves FC, et al. LXR/ApoE Activation Restricts Innate Immune Suppression in Cancer. Cell. (2018), 172:825-840 e818.

8. Shi Y, Holtzman DM. Interplay between innate immunity and Alzheimer disease: APOE and TREM2 in the spotlight. Nat Rev Immunol. (2018), 18:759-772.

9. Geng H, Law PP, Ng MC, Li T, Liang LY, Ge TF, et al. APOE genotype-function relationship: evidence of -491 A/T promoter polymorphism modifying transcription control but not type 2 diabetes risk. PLoS One. (2011), 6:e24669. 
10. Sakashita K, Tanaka F, Zhang X, Mimori K, Kamohara Y, Inoue H, Sawada T, Hirakawa K, Mori M. Clinical significance of ApoE expression in human gastric cancer. Oncol Rep. (2008), 20:1313-1319

11. Rhodes DR, Yu J, Shanker K, Deshpande N, Varambally R, Ghosh D, Barrette T, Pandey A, Chinnaiyan AM. ONCOMINE: a cancer microarray database and integrated data-mining platform. Neoplasia. (2004), 6:1-6.

12. Asplund A, Edqvist PH, Schwenk JM, Ponten F. Antibodies for profiling the human proteome-The Human Protein Atlas as a resource for cancer research. Proteomics. (2012), 12:2067-2077.

13. Chandrashekar DS, Bashel B, Balasubramanya SAH, Creighton CJ, Ponce-Rodriguez I, Chakravarthi B, Varambally S. UALCAN: A Portal for Facilitating Tumor Subgroup Gene Expression and Survival Analyses. Neoplasia. (2017), 19:649-658.

14. Szklarczyk D, Gable AL, Lyon D, Junge A, Wyder S, Huerta-Cepas J, et al. STRING v11: protein-protein association networks with increased coverage, supporting functional discovery in genome-wide experimental datasets. Nucleic Acids Res. (2019), 47:D607-D613.

15. Warde-Farley D, Donaldson SL, Comes O, Zuberi K, Badrawi R, Chao P, et al. The GeneMANIA prediction server: biological network integration for gene prioritization and predicting gene function. Nucleic Acids Res. (2010), 38:W214-220.

16. Vasaikar SV, Straub P, Wang J, Zhang B. LinkedOmics: analyzing multi-omics data within and across 32 cancer types. Nucleic Acids Res. (2018), 46:D956-D963.

17. Liu CJ, Hu FF, Xia MX, Han L, Zhang Q, Guo AY. GSCALite: a web server for gene set cancer analysis. Bioinformatics. (2018), 34:3771-3772.

18. Li T, Fan J, Wang B, Traugh N, Chen Q, Liu JS, Li B, Liu XS. TIMER: A Web Server for Comprehensive Analysis of Tumor-Infiltrating Immune Cells. Cancer Res. (2017), 77:e108-e110.

19. Danaher P, Warren S, Dennis L, D'Amico L, White A, Disis ML, et al. Gene expression markers of Tumor Infiltrating Leukocytes. J Immunother Cancer. (2017), 5:18.

20. Sousa S, Maatta J. The role of tumour-associated macrophages in bone metastasis. J Bone Oncol. (2016), 5:135-138.

21. Ru B, Wong CN, Tong Y, Zhong JY, Zhong SSW, Wu WC, et al. TISIDB: an integrated repository portal for tumor-immune system interactions. Bioinformatics. (2019), 35:4200-4202.

22. Remmele W, Stegner HE. [Recommendation for uniform definition of an immunoreactive score (IRS) for immunohistochemical estrogen receptor detection (ER-ICA) in breast cancer tissue]. Pathologe. (1987), 8:138-140

23. Chan AW, Gill RS, Schiller D, Sawyer MB. Potential role of metabolomics in diagnosis and surveillance of gastric cancer. World J Gastroenterol. (2014), 20:12874-12882.

24. Koleck TA, Bender CM, Sereika SM, Ahrendt G, Jankowitz RC, McGuire KP, Ryan CM, Conley YP. Apolipoprotein E genotype and cognitive function in postmenopausal women with early-stage breast cancer. Oncol Nurs Forum. (2014), 41:E313-325. 
25. Calabuig-Navarro MV, Jackson KG, Walden CM, Minihane AM, Lovegrove JA. Apolipoprotein E genotype has a modest impact on the postprandial plasma response to meals of varying fat composition in healthy men in a randomized controlled trial. J Nutr. (2014), 144:17751780.doi:10.3945/jn.114.197244

26. Su WP, Chen YT, Lai WW, Lin CC, Yan JJ, Su WC. Apolipoprotein E expression promotes lung adenocarcinoma proliferation and migration and as a potential survival marker in lung cancer. Lung Cancer. (2011), 71:28-33.

27. Chen YC, Pohl G, Wang TL, Morin PJ, Risberg B, Kristensen GB, Yu A, Davidson B, Shih le M. Apolipoprotein $E$ is required for cell proliferation and survival in ovarian cancer. Cancer Res. (2005), 65:331-337

28. Xu X, Wan J, Yuan L, Ba J, Feng P, Long W, et al. Serum levels of apolipoprotein E correlates with disease progression and poor prognosis in breast cancer. Tumour Biol. (2016).

29. Ben Hassen C, Gutierrez-Pajares JL, Guimaraes C, Guibon R, Pinault M, Fromont G, Frank PG. Apolipoprotein-mediated regulation of lipid metabolism induces distinctive effects in different types of breast cancer cells. Breast Cancer Res. (2020), 22:38.

30. Bouris P, Skandalis SS, Piperigkou Z, Afratis N, Karamanou K, Aletras AJ, Moustakas A, Theocharis AD, Karamanos NK. Estrogen receptor alpha mediates epithelial to mesenchymal transition, expression of specific matrix effectors and functional properties of breast cancer cells. Matrix Biol. (2015), 43:42-60.

31. Jiao Q, Bi L, Ren Y, Song S, Wang Q, Wang YS. Advances in studies of tyrosine kinase inhibitors and their acquired resistance. Mol Cancer. (2018), 17:36.

32. Lu TX, Rothenberg ME. MicroRNA. J Allergy Clin Immunol. (2018), 141:1202-1207.

33. Lambert SA, Jolma A, Campitelli LF, Das PK, Yin Y, Albu M, et al. The Human Transcription Factors. Cell. (2018), 172:650-665.

34. Krasemann S, Madore C, Cialic R, Baufeld C, Calcagno N, El Fatimy R, et al. The TREM2-APOE Pathway Drives the Transcriptional Phenotype of Dysfunctional Microglia in Neurodegenerative Diseases. Immunity. (2017), 47:566-581 e569.

35. Nam KN, Wolfe CM, Fitz NF, Letronne F, Castranio EL, Mounier A, Schug J, Lefterov I, Koldamova R. Integrated approach reveals diet, APOE genotype and sex affect immune response in APP mice. Biochim Biophys Acta Mol Basis Dis. (2018), 1864:152-161.

\section{Tables}

TABLE 1 APOE expression and clinicopathological characteristics of 58 specimens in Shanghai corhort 


\begin{tabular}{|c|c|c|c|c|c|c|}
\hline \multirow[t]{2}{*}{ Characteristics } & \multirow[t]{2}{*}{ Total } & \multicolumn{2}{|c|}{ APOE expression } & \multirow[t]{2}{*}{$P$ value } & \multirow[t]{2}{*}{$\chi \square$ value } & \multirow{2}{*}{$\begin{array}{l}\text { Correlation } \\
\text { rvalue }\end{array}$} \\
\hline & & Low & High & & & \\
\hline Age (years) & & & & 0.735 & 0.11 & \\
\hline$<45$ & 24 & 13 & 11 & & & \\
\hline$\geq 45$ & 32 & 18 & 16 & & & \\
\hline Gender & & & & 0.974 & 0.01 & \\
\hline Female & 13 & 7 & 6 & & & \\
\hline Male & 45 & 24 & 21 & & & \\
\hline Pathological stage & & & & 0.001 & 13.726 & 0.6978 \\
\hline Low $(I+I I)$ & 37 & 26 & 11 & & & \\
\hline High (III +IV) & 21 & 5 & 16 & & & \\
\hline Lymph node metastasis & & & & 0.001 & 14.09 & 0.7 \\
\hline Negative & 32 & 25 & 7 & & & \\
\hline Positive & 26 & 6 & 20 & & & \\
\hline
\end{tabular}

Statistical analyses were performed by the Pearson $\chi \llbracket$ test

TABLE 2. The Kinase, miRNA and transcription factor-target networks of APOE in Thyroid carcinoma (LinkedOmics). 


\begin{tabular}{|llll|}
\hline Enriched Category & Geneset & LeadingEdgeNum & FDR \\
\hline Kinase Target & Kinase_LCK & 43 & \\
\hline miRNA Target & & & 0.042960 \\
& TAATGTG,MIR-323 & 56 & 0.017515 \\
Transcription Factor Target & & & \\
& & & \\
& V\$SAP1_C & 84 & 0.010616 \\
& V\$STAT5B_01 & 75 & 0.014154 \\
& V\$NERF_Q2 & 79 & 0.014154 \\
& V\$NFKAPPAB65_01 & 95 & 0.015974 \\
& RGAGGAARY_V\$PU1_Q6 & 145 & 0.01607 \\
& V\$BACH2_01 & 73 & 0.016728 \\
& V\$NGFIC_01 & 57 & 0.018137 \\
& V\$SBP1_Q6 & 53 & 0.01859 \\
\hline
\end{tabular}

TABLE 3. Multivariate analyses of APOE expressions and immune cells related to overall survival in PTC. 


\begin{tabular}{|llllll|}
\hline & coef & HR & $95 \%$ Cl_I & $95 \% \mathrm{Cl} \_u$ & p.value \\
\hline Age & 0.213 & 1.238 & 1.119 & $1.369000 \mathrm{e}+00$ & $\mathbf{0 . 0 0 0}$ \\
\hline gendermale & 0.155 & 1.168 & 0.264 & $5.173000 \mathrm{e}+00$ & 0.838 \\
\hline raceBlack & 16.500 & 14650401.817 & 0.000 & Inf & 0.999 \\
\hline raceWhite & 15.525 & 5524187.430 & 0.000 & Inf & 0.999 \\
\hline Purity & 6.069 & 432.136 & 11.001 & $1.697427 \mathrm{e}+04$ & $\mathbf{0 . 0 0 1}$ \\
\hline B_cell & 0.852 & 2.344 & 0.000 & $4.079308 \mathrm{e}+05$ & 0.890 \\
\hline CD8_Tcell & -20.499 & 0.000 & 0.000 & $1.110000 \mathrm{e}-01$ & $\mathbf{0 . 0 2 8}$ \\
\hline CD4_Tcell & 9.396 & 12044.886 & 0.240 & $6.037987 \mathrm{e}+08$ & 0.089 \\
\hline Macrophage & -32.041 & 0.000 & 0.000 & $1.380000 \mathrm{e}-01$ & $\mathbf{0 . 0 3 7}$ \\
\hline Neutrophil & -50.757 & 0.000 & 0.000 & $1.296886 \mathrm{e}+08$ & 0.152 \\
\hline Dendritic & 15.953 & 8478449.036 & 0.813 & $8.844267 \mathrm{e}+13$ & $\mathbf{0 . 0 4 3}$ \\
\hline APOE & -0.393 & 0.675 & 0.442 & $1.030000 \mathrm{e}+00$ & 0.069 \\
\hline
\end{tabular}

TABLE 4. Correlation between APOE and related gene markers of relevant immune cells. 


\begin{tabular}{|c|c|c|c|c|c|}
\hline \multirow[t]{2}{*}{ Immune cell } & \multirow[t]{2}{*}{ Gene Markers } & \multicolumn{2}{|l|}{ None } & \multicolumn{2}{|l|}{ Purity } \\
\hline & & $r$ & $\mathrm{p}$ & $r$ & $\mathrm{p}$ \\
\hline \multirow[t]{2}{*}{ CD8+T cell } & CD8A & 0.21 & $1.87 \mathrm{e}-06$ & 0.209 & $3.37 e-06$ \\
\hline & CD8B & 0.385 & $0 \mathrm{E}+00$ & 0.388 & $5.02-19$ \\
\hline \multirow[t]{3}{*}{ TAM } & CCL2 & 0.233 & $1.13 e-07$ & 0.248 & $2.88 \mathrm{e}-08$ \\
\hline & CD68 & 0.29 & $3.14 \mathrm{e}-11$ & 0.304 & $6.73 e-12$ \\
\hline & IL10 & 0.202 & $4.4 \mathrm{e}-06$ & 0.217 & $1.31 \mathrm{e}-06$ \\
\hline \multirow[t]{3}{*}{ M1 Macrophage } & INOS(NOS2) & 0.114 & $1.02 \mathrm{e}-02$ & 0.109 & $1.63 \mathrm{e}-02$ \\
\hline & IRF5 & 0.367 & $2.92 e-18$ & 0.367 & $5.48 \mathrm{e}-17$ \\
\hline & COX2(PTGS2) & 0.156 & $4.11 \mathrm{e}-04$ & 0.171 & $1.46 \mathrm{e}-04$ \\
\hline \multirow[t]{3}{*}{ M2 Macrophage } & CD163 & 0.299 & $6.9 \mathrm{e}-12$ & 0.313 & $1.48 \mathrm{e}-12$ \\
\hline & VSIG4 & 0.239 & $5.17-08$ & 0.24 & $7.91 \mathrm{e} 08$ \\
\hline & MS4A4A & 0.31 & $1.17 \mathrm{e}-12$ & 0.318 & $6.14 \mathrm{e}-13$ \\
\hline
\end{tabular}

Figures 


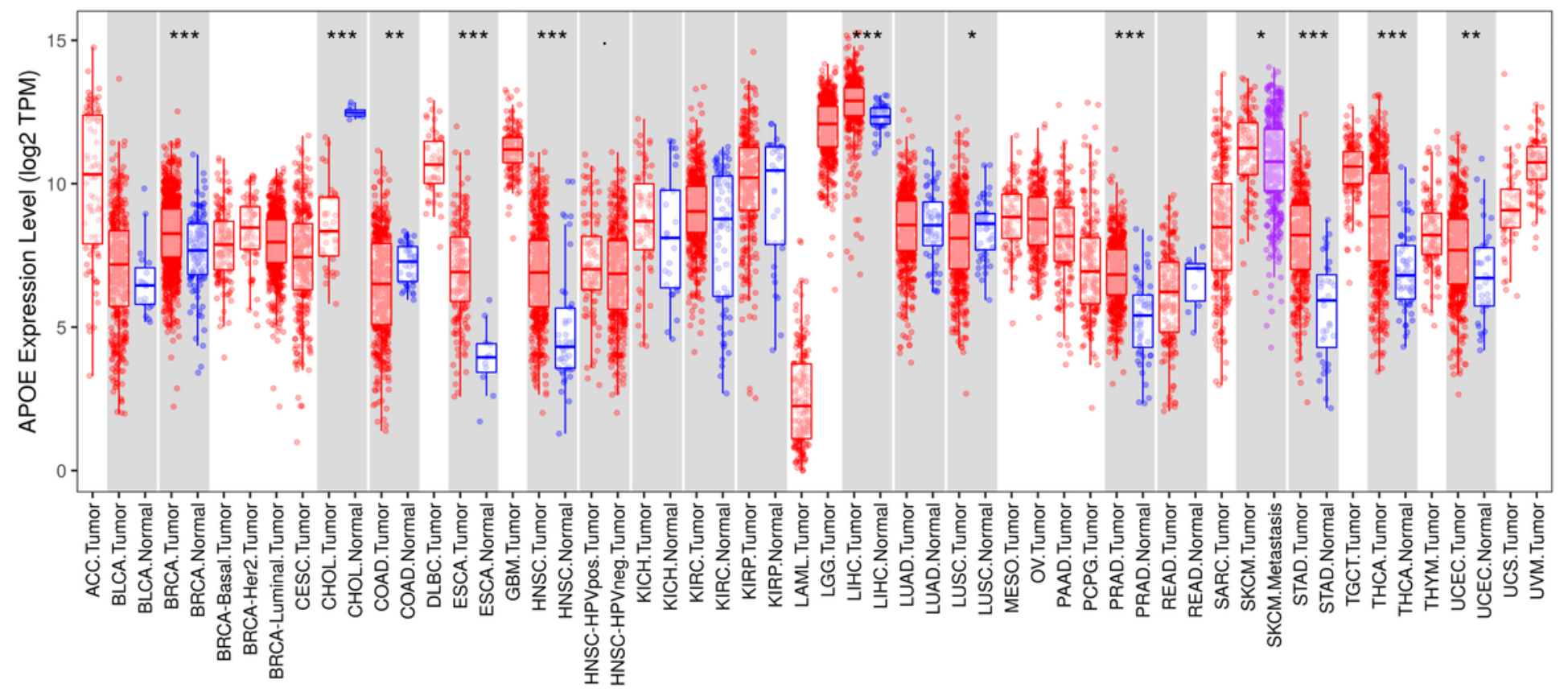

Figure 1

APOE expression in different types of human cancers (TIMER). Note: ${ }^{\star} P<0.05,{ }^{\star} \times P<0.01,{ }^{\star} * * P<0.001$. 


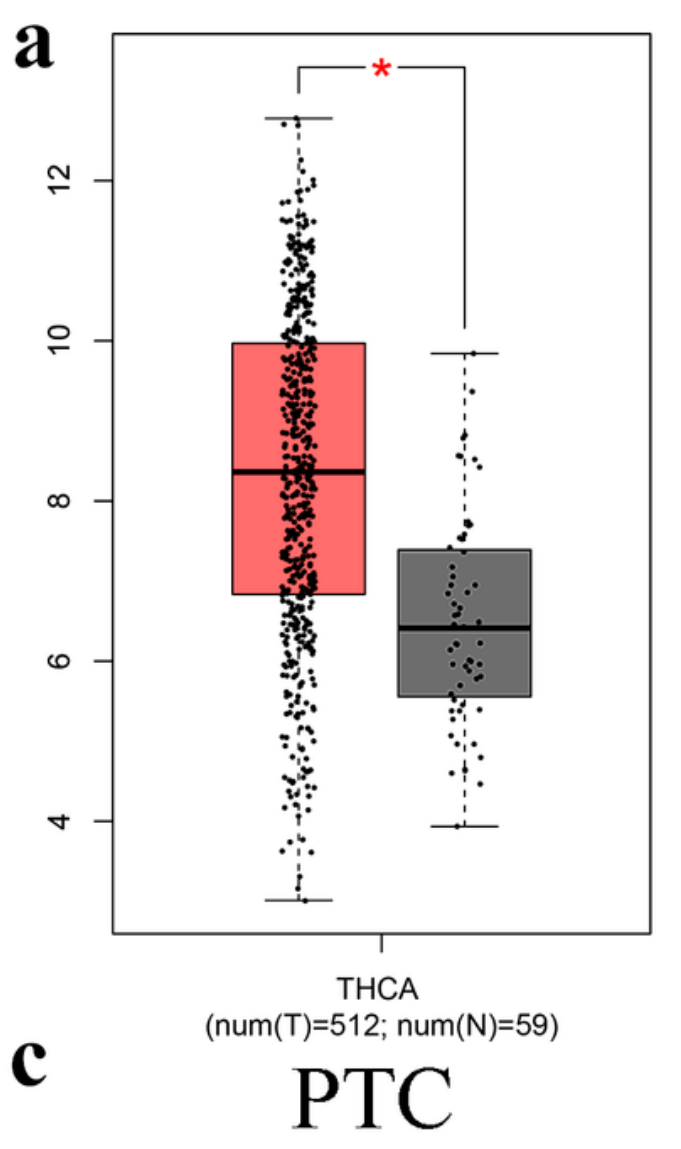

b

$$
\begin{aligned}
& \text { APOE Expression in He Thyroid } \\
& \text { Thyroid Gland Papillary Carcinoma vs. Normal }
\end{aligned}
$$

He Thyroid Statistics

Over-expression Gene Rank: 207 (in top 2\%) P-value: 2.46E-5

Reporter: 203382_s_at - t-Test: 6.363

Fold Change: 4.268

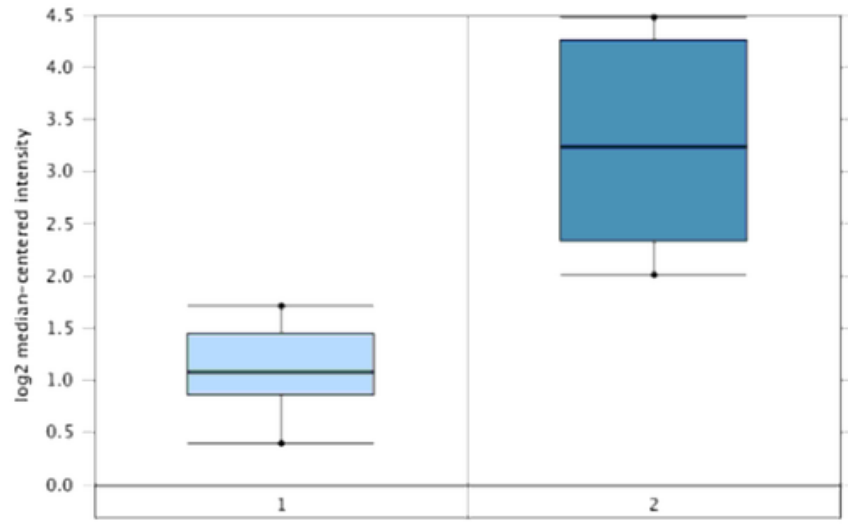

Legend

1. Thyroid Gland (9)

2. Thyroid Gland Papillary Carcinoma

(9)

\section{adjacent tissue}

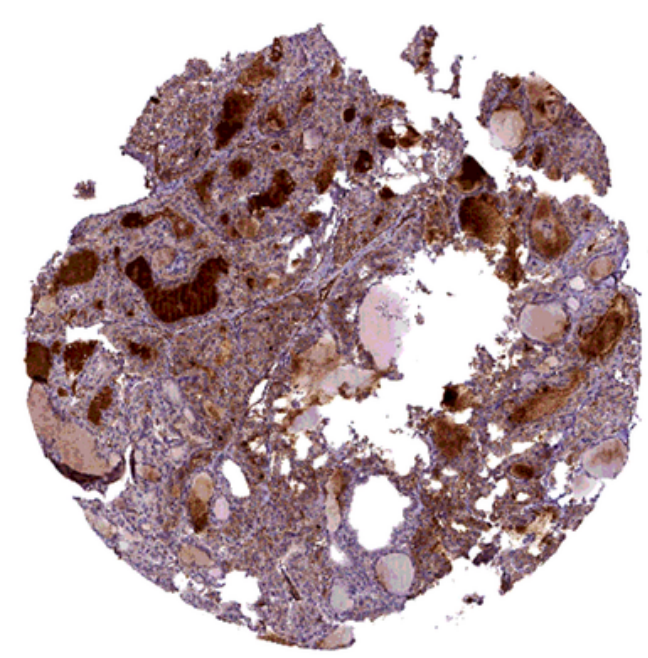

staining: Moderate

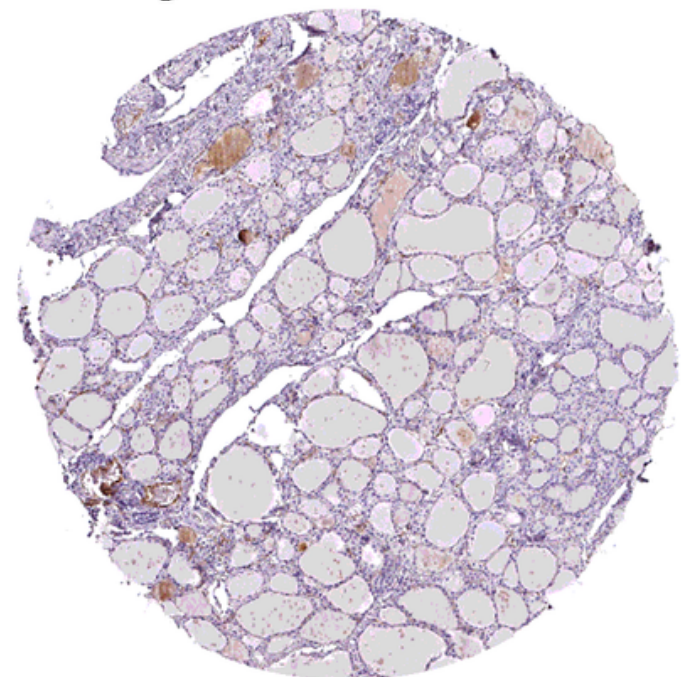

staining: Not detect

Antibody CAB008363

Figure 2

APOE expression in PTC. a Box plot comparing APOE mRNA expression in thyroid specimens from 512 patients with PTC (red) and 59 normal controls (black) from TCGA. b Box plot showing APOE mRNA levels in PTC specimens (dark blue) and normal controls (light blue) according to datasets from $\mathrm{He}$ Thyroid. c APOE immunohistochemical staining results of PTC and adjacent tissues according to the HPA. 
a Expression of APOE in THCA based on patient's gender

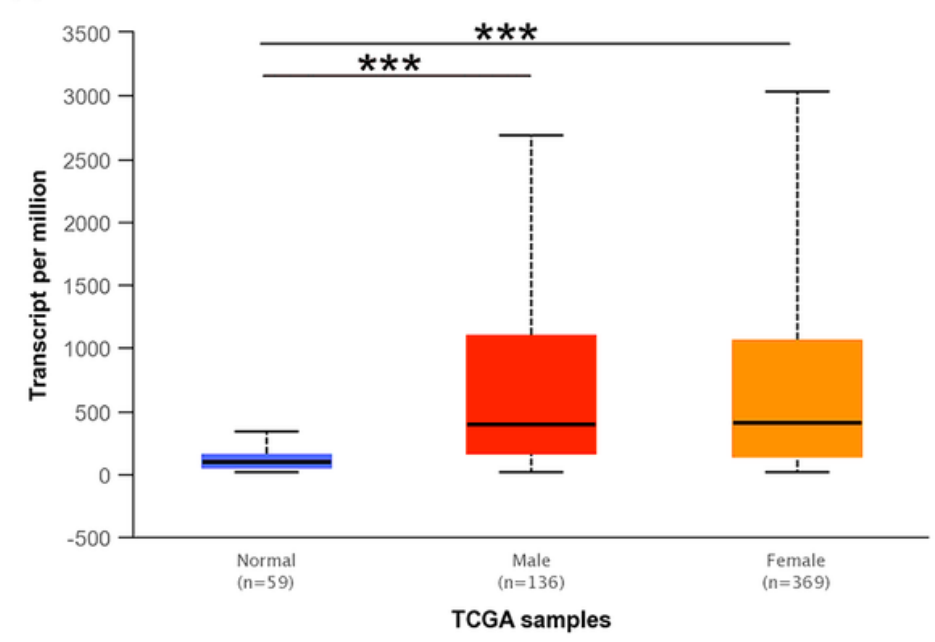

Expression of APOE in THCA based on nodal metastasis

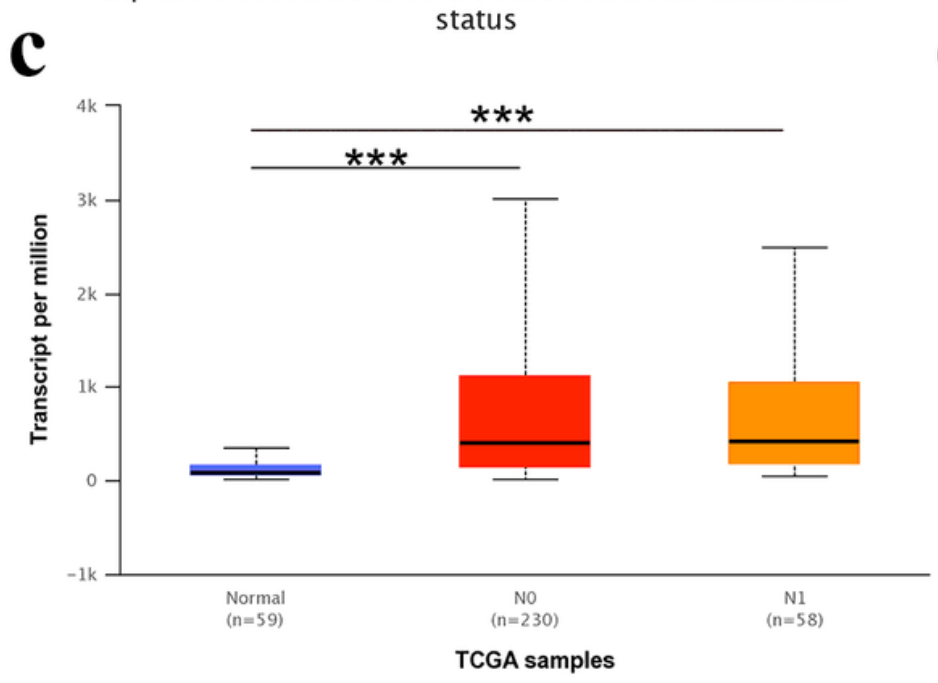

D Expression of APOE in THCA based on patient's age

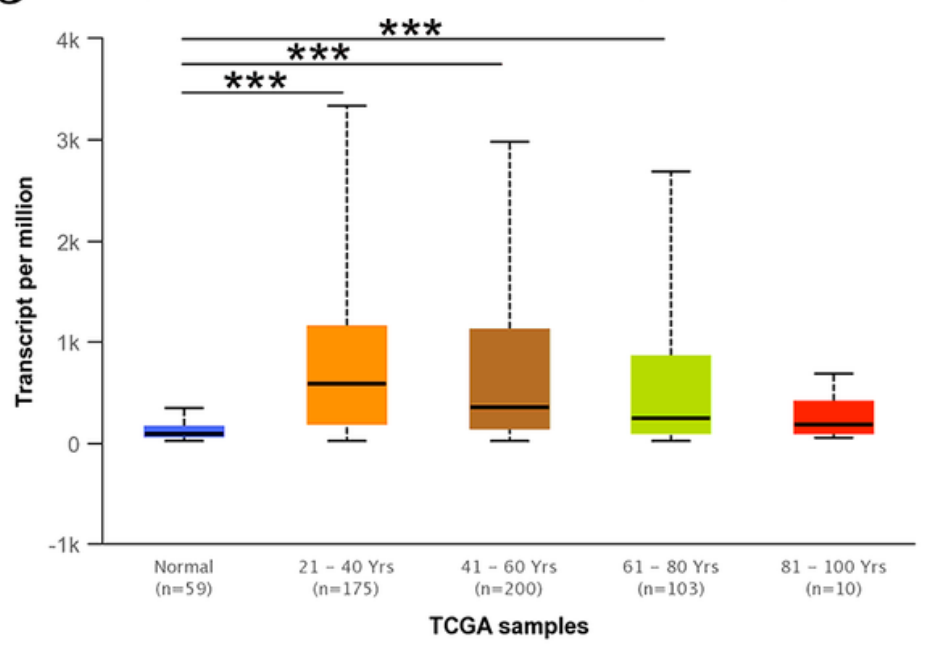

Expression of APOE in THCA based on individual cancer

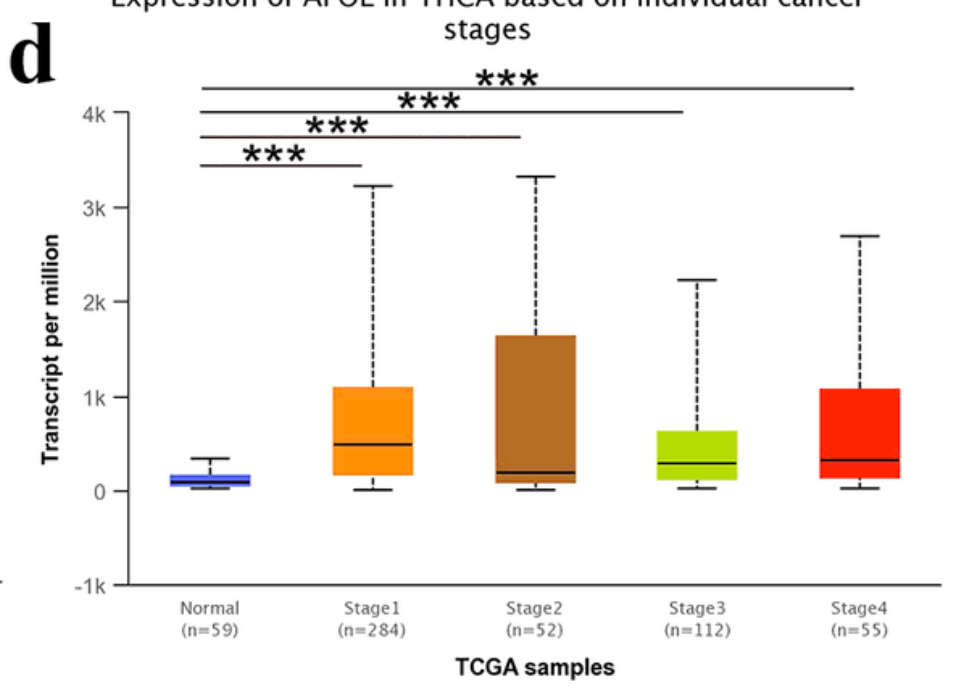

Figure 3

APOE transcription in subgroups of patients with PTC. a Boxplot showing relative expression of APOE based on patient's gender. b Boxplot showing relative expression of APOE based on patient's age. $c$ Boxplot showing relative expression of APOE based on nodal metastasis status. d Boxplot showing relative expression of APOE in thyroid cancer based on individual cancer stages. 

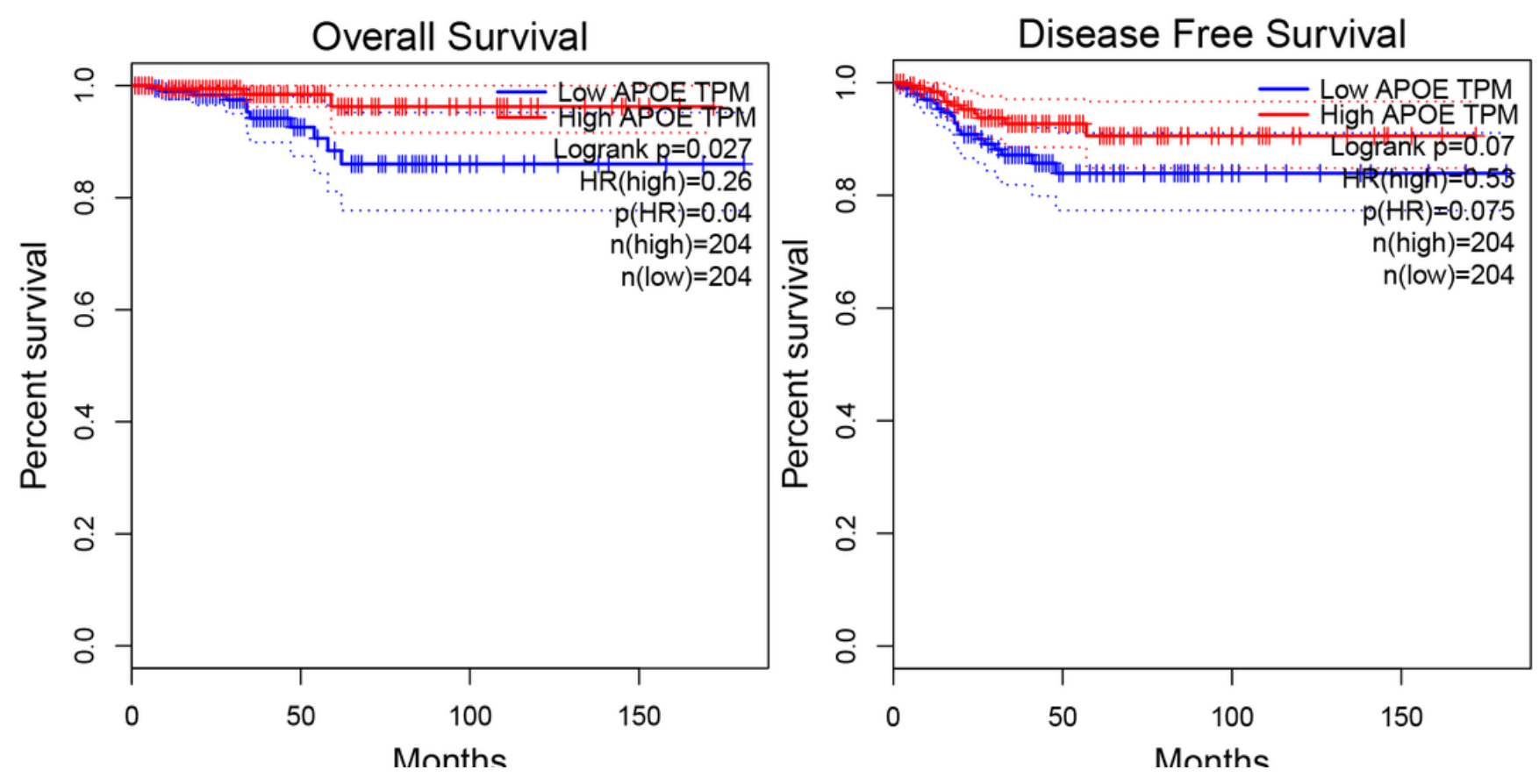

Figure 4

The overall survival and disease-free survival curve of APOE in PTC 
a

ROC of APOE in TCGA cohort

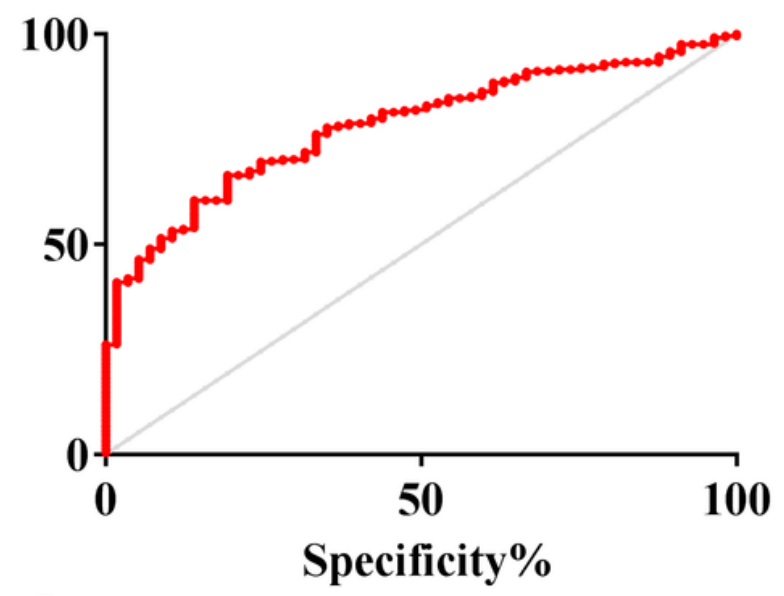

b

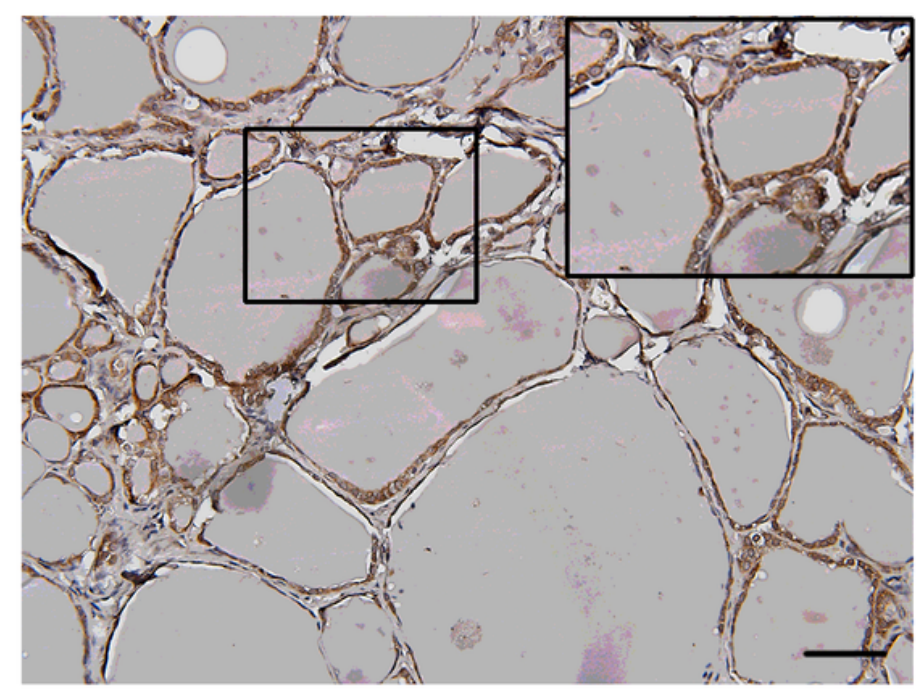

Normal tissue
ROC of APOE in Shanghai cohort
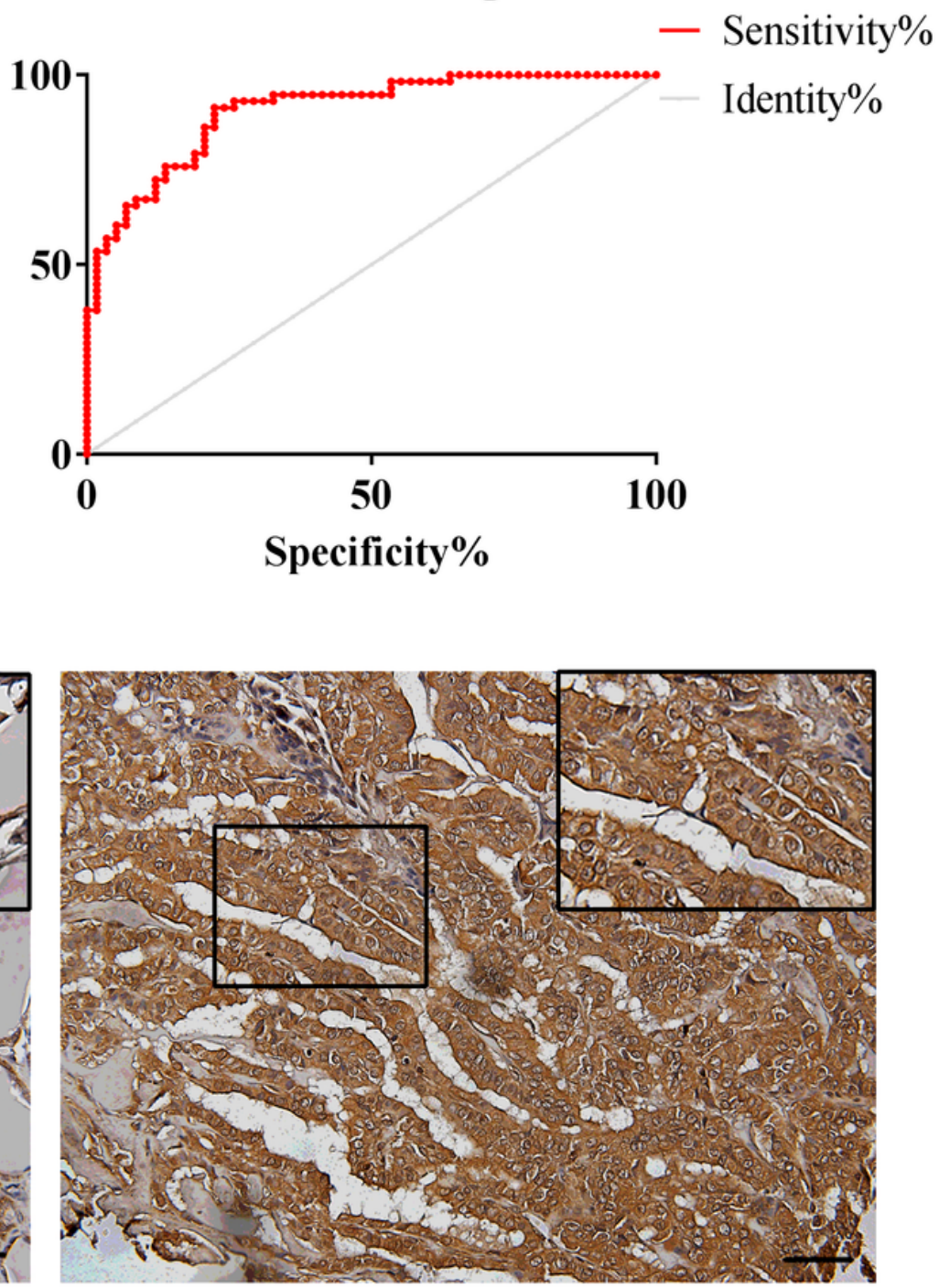

PTC tissue

Figure 5

The ROC curve and immunohistochemical staining results of APOE in TCGA cohort and Shanghai cohort . a The ROC curve of TCGA cohort and Shanghai cohort. APOE in TCGA cohort, AUC $=0.7819(95 \% \mathrm{Cl}$ : 0.7314-0.8324), $p<0.0001$; APOE in Shanghai cohort AUC=0.9064 (95\% Cl: 0.8546-0.9581), $p<0.0001 ; b$ The immunohistochemical staining results of APOE of Shanghai cohort. APOE proteins were higher in PTC tissues, compared to tumor-adjacent tissues, bar $=50 \mu \mathrm{m}$. 


\section{a}
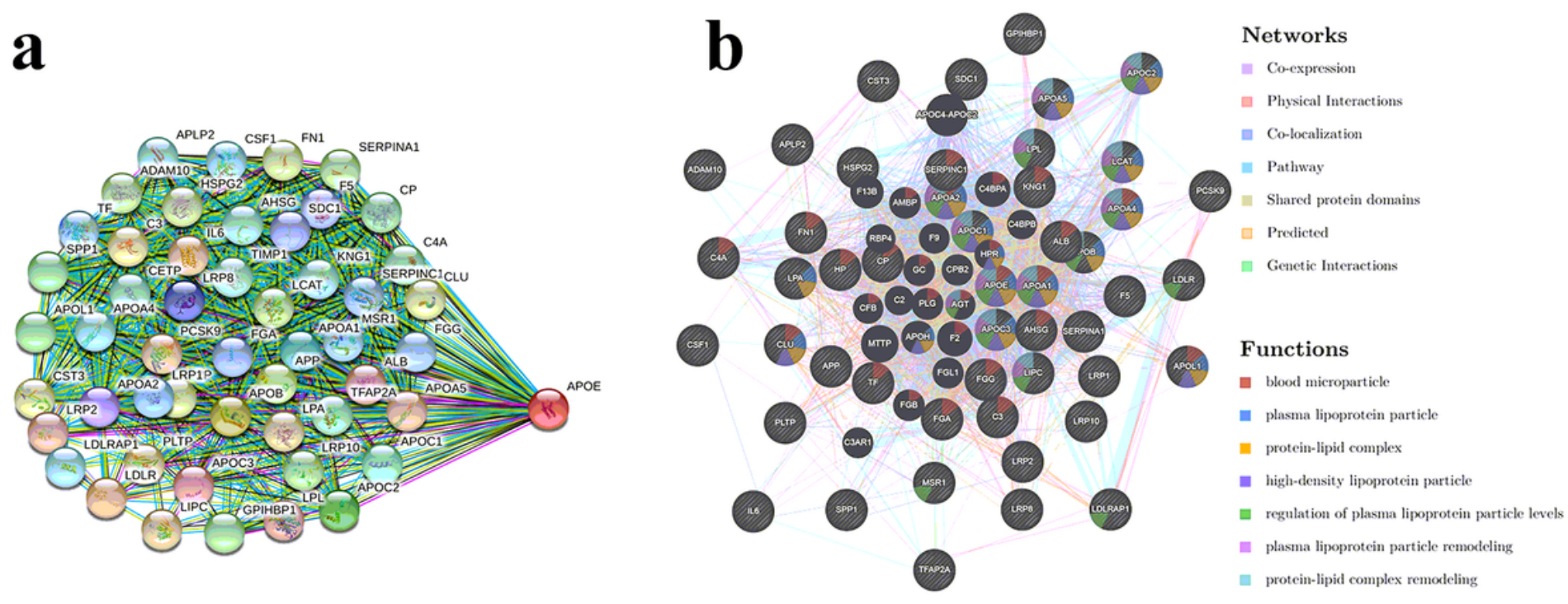

Figure 6

Neighbor gene network of differently expressed APOE in PTC patients. a Neighbor gene network of APOE of STRING. b Protein-protein interaction network of APOE in GeneMANIA. 

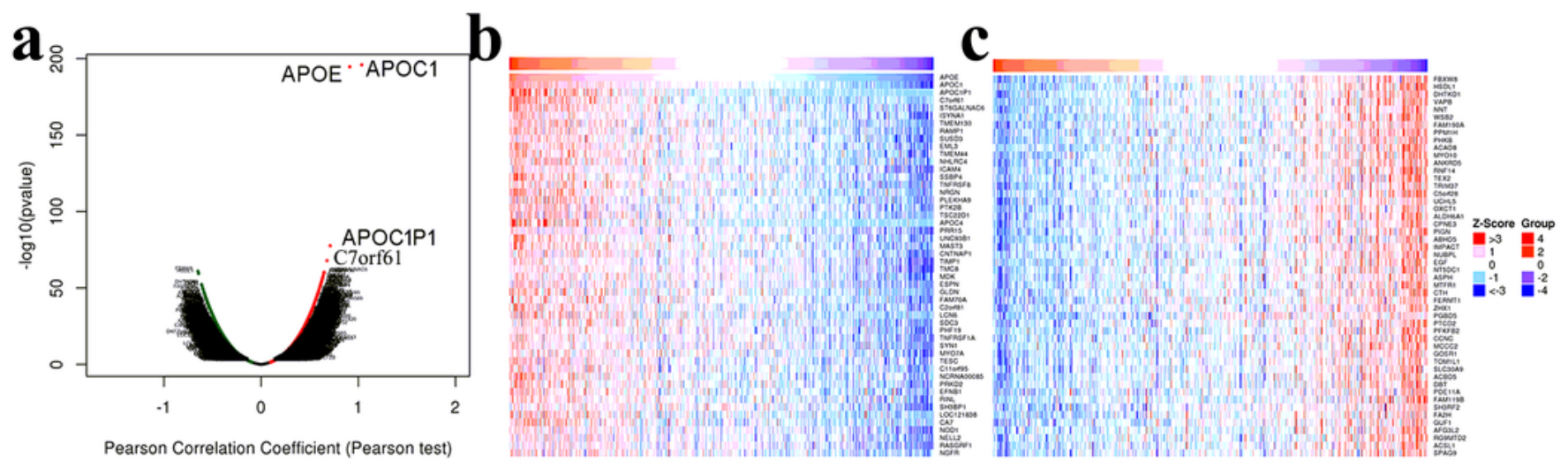

Pearson Correlation Coefficient (Pearson test)
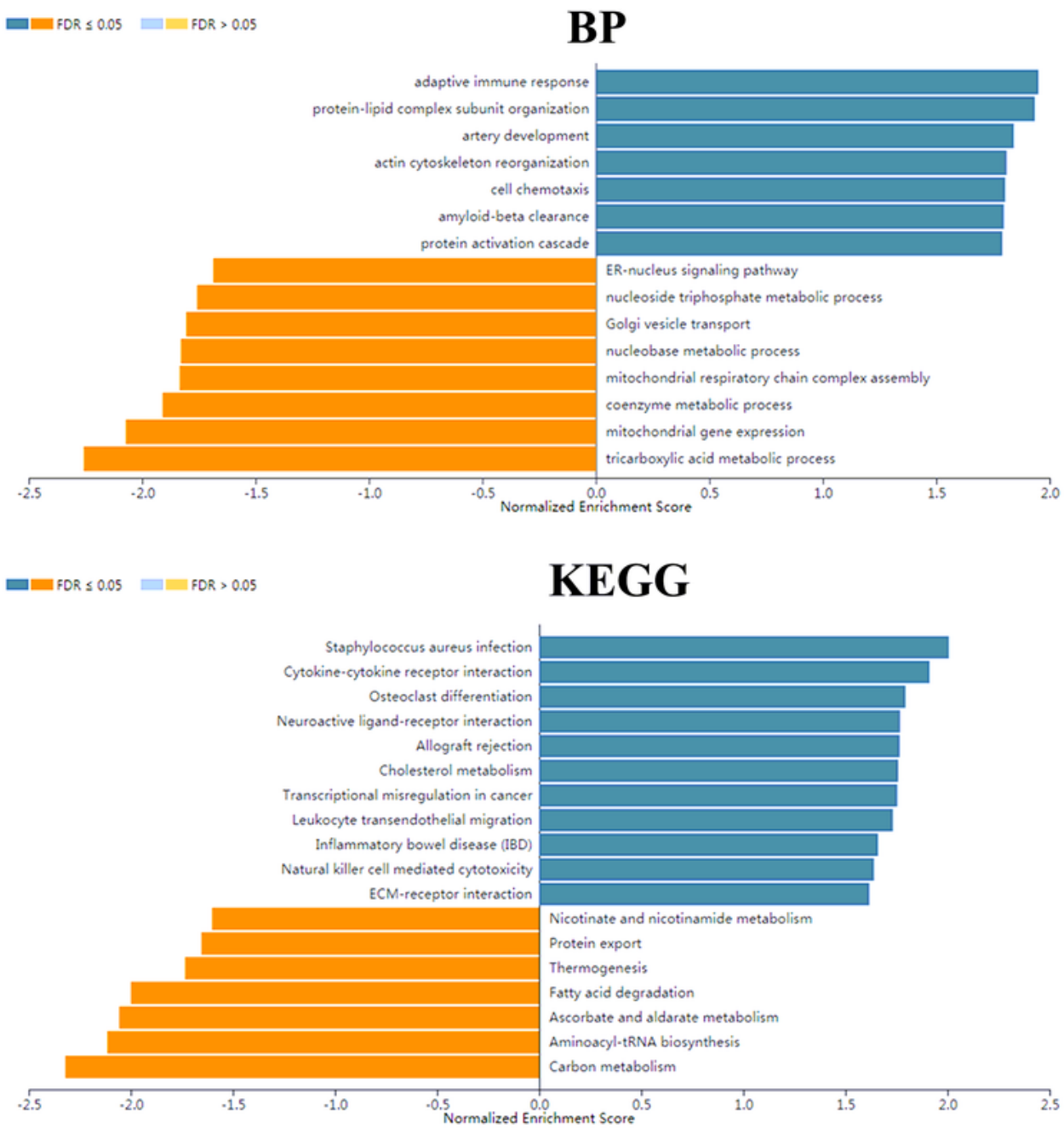

\section{Figure 7}

APOE co-expression genes in PTC (LinkedOmics). a The genes positively and negatively correlated with APOE in PTC. $b$ The top-50 gene positively correlated with APOE in PTC in heat maps. $c$ The top-50 gene negatively correlated with APOE in PTC in heat maps. d Biological process analyse and KEGG pathways of APOE in TCGA cohort. 


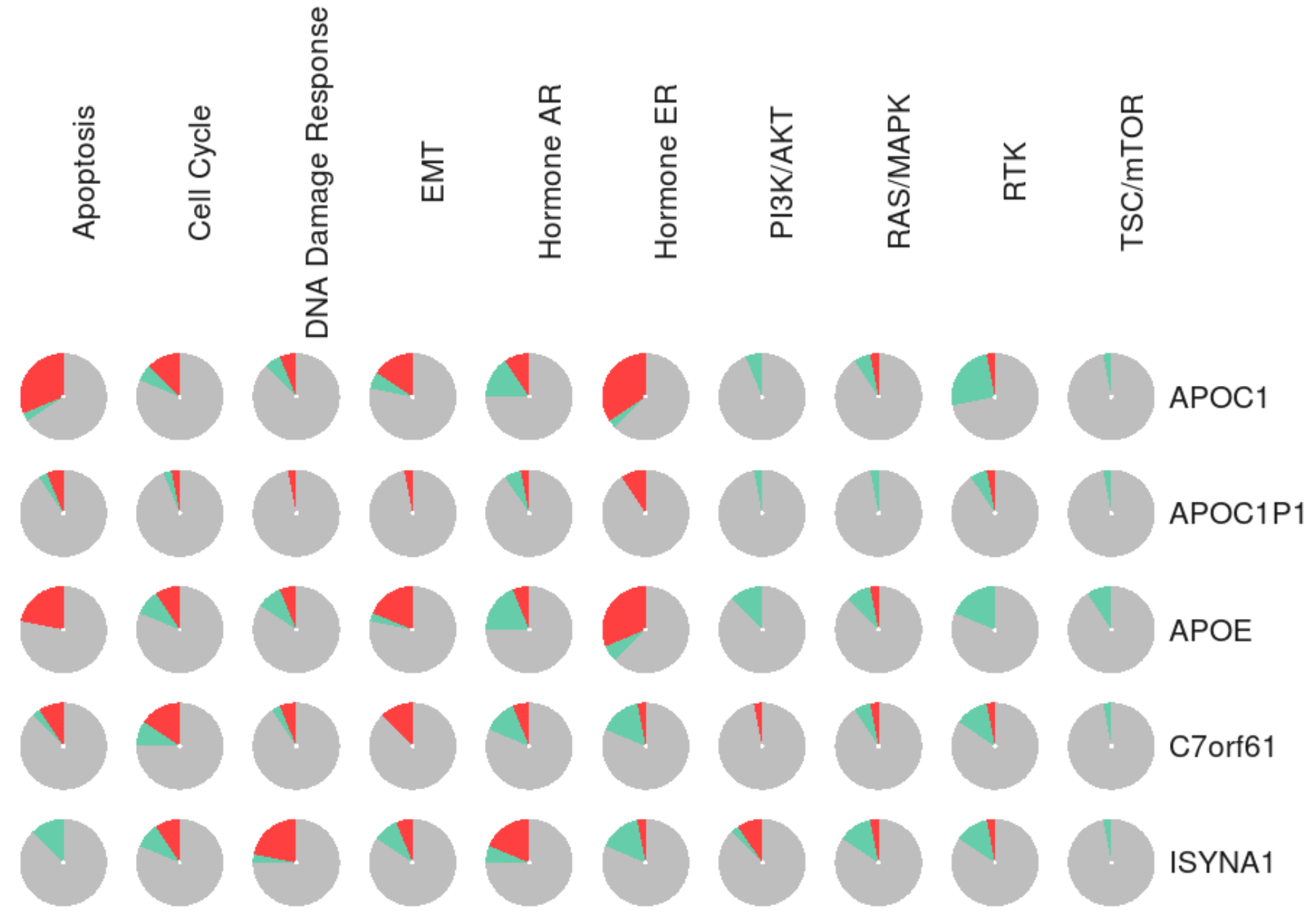

Activation Inhibition None

Figure 8

The role of APOE in the cancer related pathways (GSCALite). 
$\mathbf{a}$
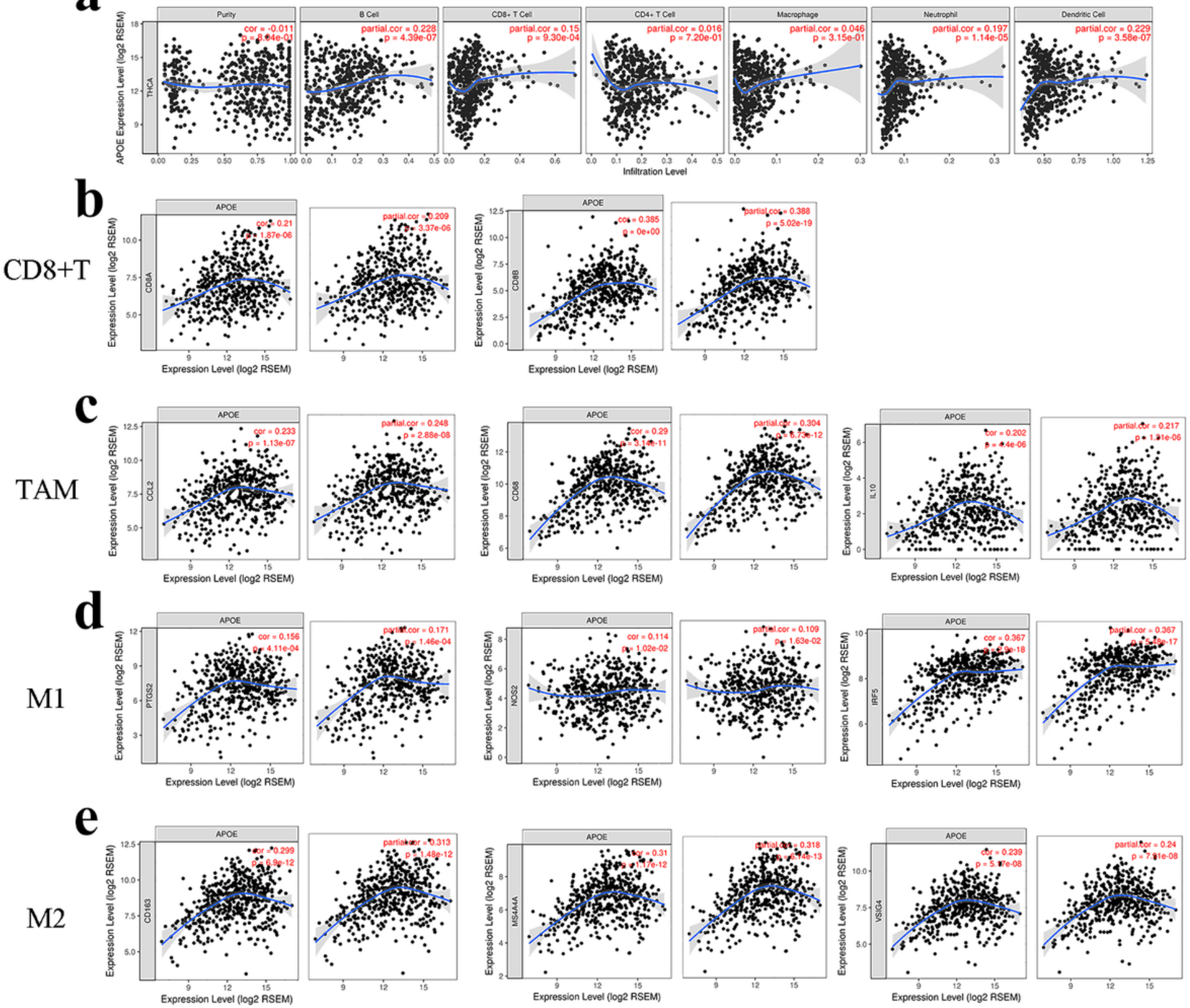

Figure 9

Correlation of APOE expression with immune cell and biomarkers in thyroid cancer. a APOE expression was positively correlated with $B$ cells (Cor $=0.228, P=4.39 \mathrm{e}-07$ ), $C D 8+T$ cells(Cor $=0.15, P=9.30 \mathrm{e}-4$ ), Neutrophils (Cor=0.197, $\mathrm{P}=1.14 \mathrm{e}-05$ ) and Dendritic cells(Cor=0.229, $\mathrm{P}=3.58 \mathrm{e}-07$ ). $\mathrm{b}$ The APOE expression was positively correlated with immune-related markers in PTC, such as CD8+T cell, TAM, M1 and M2 macrophage. 

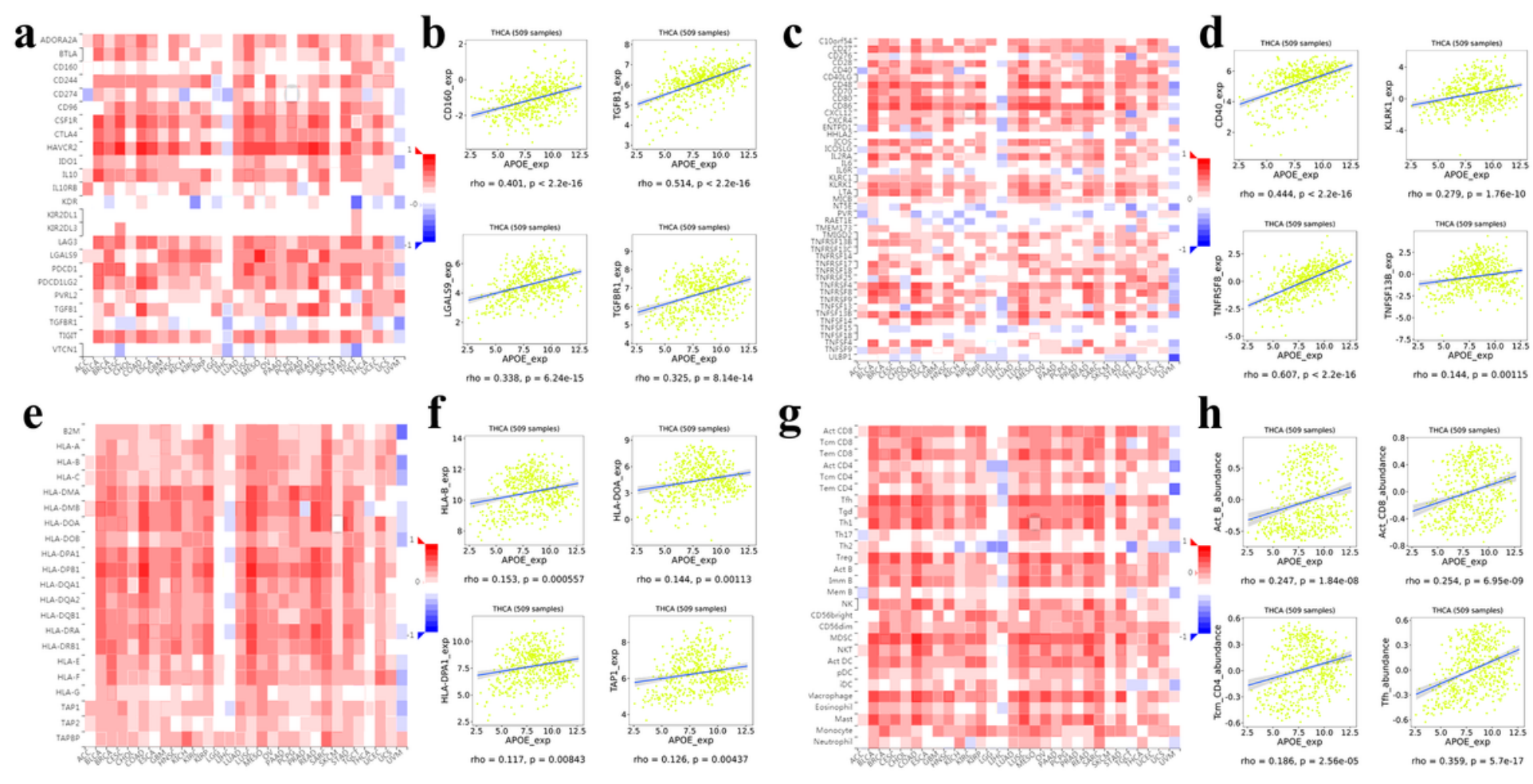

g

h
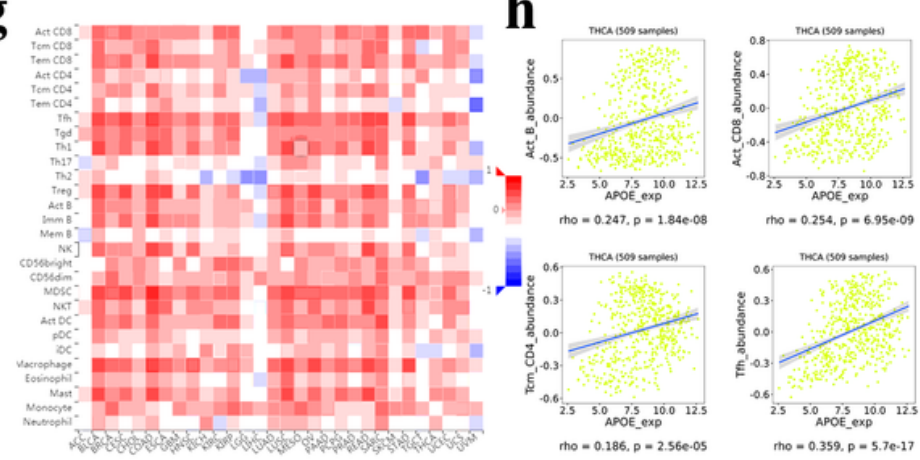

Figure 10

Spearman's correlation of APOE with lymphocytes and immunomodulators (TISIDB). a Relations between the immunoinhibitors and APOE expression. b 4 immunoinhibitors were correlated with APOE expression. c Relations between the immunostimulators and APOE expression. $\mathrm{d} 4$ immunostimulators were correlated with APOE expression. e Relations between the MHC molecules and APOE expression. $\mathrm{f} 4 \mathrm{MHC}$ molecules were correlated with APOE expression. g Relations between the tumor-infiltrating lymphocytes and APOE expression. h 4 tumor-infiltrating lymphocytes were correlated with APOE expression.
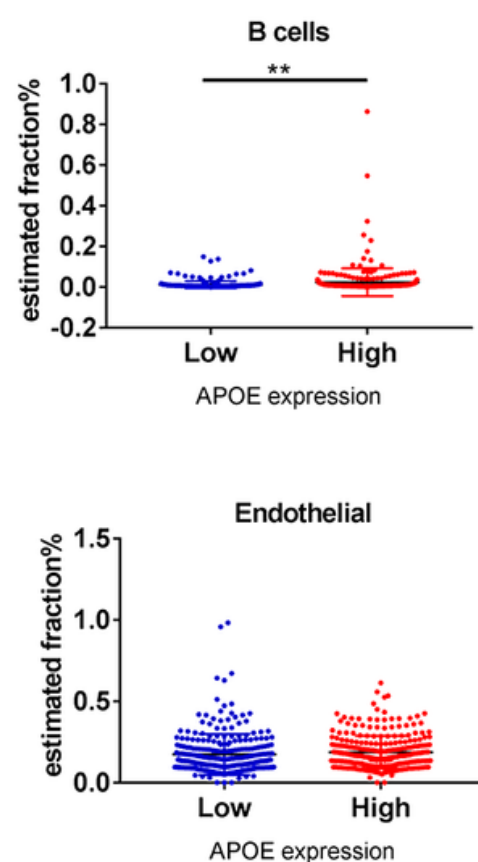

APOE expression
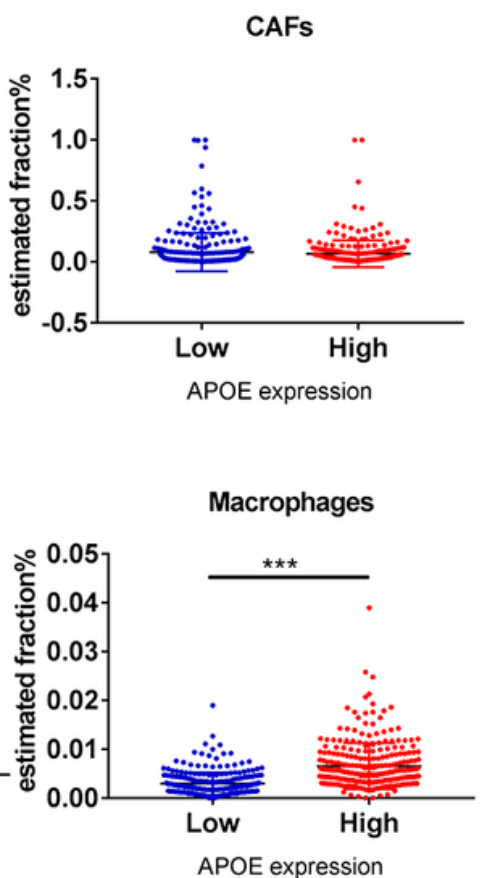
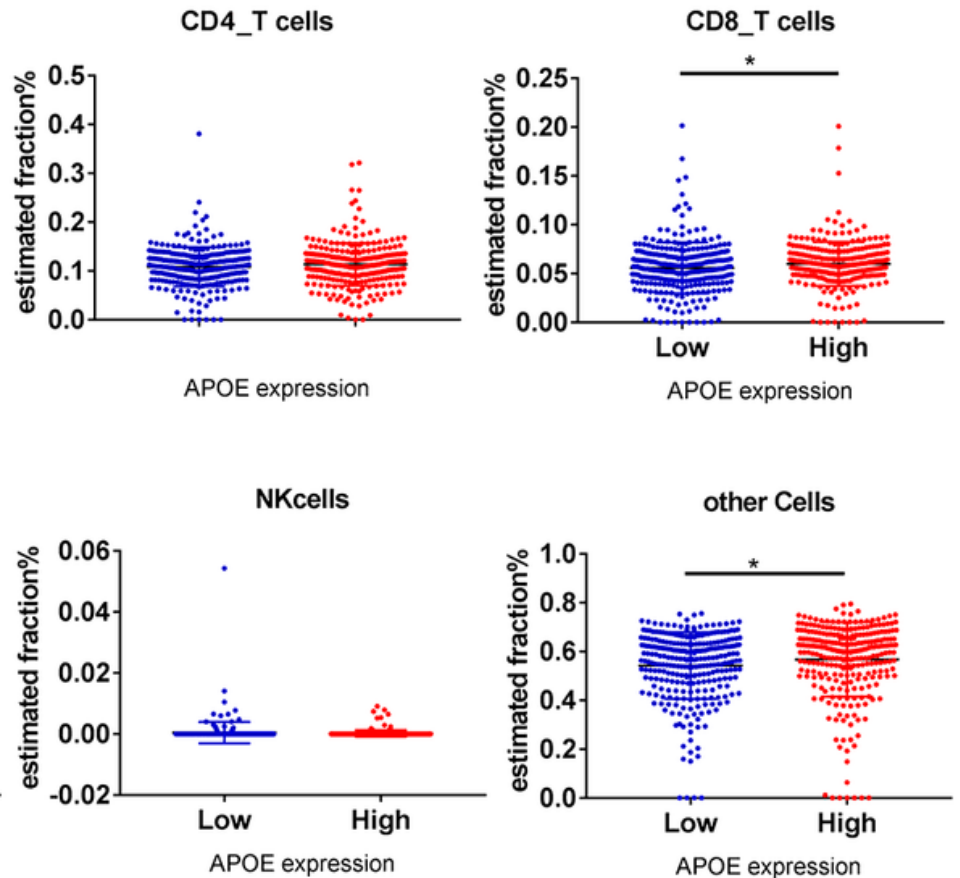


\section{Figure 11}

B cells, CD8_T cells, macrophages and other cells relative expression in APOE low group and APOE high group analyzing by online tools EPIC. Note: ${ }^{*} P<0.05$, ${ }^{\star \star} \mathrm{P}<0.01$, ${ }^{\star \star *} \mathrm{P}<0.001$.

\section{Supplementary Files}

This is a list of supplementary files associated with this preprint. Click to download.

- Supplementary.zip 